Check for updates

Cite this: RSC Adv., 2017, 7, 18609

\title{
Annealing effects on the magnetic properties of highly-packed vertically-aligned nickel nanotubes
}

\author{
Vlad-Andrei Antohe, (D) *ab Emeline Nysten, $\uparrow^{\mathrm{a}}$ Juan Manuel Martínez-Huerta, $\uparrow^{a}$ \\ Pedro Miguel Pereira de Sáa and Luc Piraux*a
}

\begin{abstract}
We reported an efficient way of preparing large and dense ferromagnetic arrays of vertically-aligned $\mathrm{Ni}$ nanotubes (NTs). The synthesis method allowed then for a direct, effective and controllable thermal conversion of the as-prepared Ni NTs into heterostructured Ni/NiO NTs, without compromising the nanostructures' verticality to the substrate and their initial tubular integrity. Next, we demonstrated the ability to tune the dipolar interaction field (DIF) in this array of one-dimensional (1D) $\mathrm{Ni} / \mathrm{NiO}$ heterostructures, just by slightly changing the thickness of the antiferromagnetic NiO layer. Furthermore, the proposed fabrication method assures full compatibility with the standard Si processing, providing thus a direct pathway towards large-scale integrability and manufacturing of a new generation of magnetic devices relying on dense arrays of vertical 1D magnetic nanostructures.
\end{abstract}

Received 30th January 2017 Accepted 18th March 2017

DOI: 10.1039/c7ra01276d

rsc.li/rsc-advances
Nevertheless, little has been yet reported regarding the dipolar interaction field (DIF) in dense arrays of NTs, ${ }^{25,26}$ which is expected to play an important role in the development of magnetic devices with controlled anisotropy. Furthermore, the fabrication of large arrays of ferromagnetic/antiferromagnetic NTs is of particular interest, as such heterostructured NTs can be expected to overcome the superparamagnetic limit and enhance the thermal stability of ultra-high density magnetic memory storage devices. ${ }^{27}$ However, their fabrication remains up-to-date challenging, hence a limited number of studies have been performed so far on metal oxide NT arrays directly prepared by either chemical vapor deposition (CVD), ${ }^{\mathbf{2 4}, \mathbf{2 8}-\mathbf{3 0}}$ anodization methods $\mathrm{s}^{31-34}$ or other template-free protocols. ${ }^{35}$

A cost-effective way to obtain homogenous arrays of NTs with controlled geometrical parameters and vertical ordering is a multi-step process, entailing an electrochemical synthesis of metallic NTs within a suitable nanoporous template, ${ }^{13}$ followed by their subsequent thermal conversion into metal oxide NTs. ${ }^{19}$ Although the growth of densely-packed magnetic NTs was already attempted using either track-etched polycarbonate (PC ${ }^{26}$ or selfsupported anodic aluminum oxide $(\mathrm{AAO})^{36-39}$ membranes, none of these template-based technologies managed to solve the verticality problem, and thus to enable the design of homogenous vertical free-standing NT arrays with tunable geometrical features. In contrast, supported nanoporous AAO represents an amiable nanotechnological platform for development of low aspect-ratio elongated nanostructures capable of remaining perpendicular to the substrate after the embedding host removal. ${ }^{40}$ Additionally, the process allows the subsequent thermal oxidation steps of the NTs to be carried out without taking away the AAO, thus preventing the NTs outer deformation and/or lateral volume expansion during the annealing procedures.

\footnotetext{
${ }^{a}$ Institute of Condensed Matter and Nanosciences (IMCN), Université Catholique de Louvain (UCL), B-1348 Louvain-la-Neuve, Belgium. E-mail: luc.piraux@uclouvain. be; Tel: +3210473566

${ }^{b}$ Research and Development Center for Materials and Electronic \& Optoelectronic Devices (MDEO), Faculty of Physics, University of Bucharest, 077125 BucharestMăgurele, Romania. E-mail: vlad.antohe@fizica.unibuc.ro; Tel: +40 723666907

$\dagger$ Currently at: Institut für Physik, Universität Augsburg, Germany.

\$ Currently at: Instituto de Física, Universidad Autónoma de San Luis Potosí, Mexico.
}

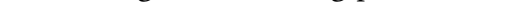


Here we report the electrochemical synthesis within supported AAO templates and subsequent controlled oxidation of vertically-aligned arrays of Ni NTs, to ultimately form $\mathrm{Ni} / \mathrm{NiO}$ heterostructured NTs with different oxide wall layer thicknesses. We found that the DIF decreases as the NiO wall thickness is increased, which shows that the oxide film can serve as a parameter for controlling the magnetic properties of the NTs, thus it could be of potential interest in applications where the dipolar interaction through the magnetic array needs to be controlled.

\section{Materials and methods}

\subsection{Samples preparation}

Analytical grade (99.99\%, unless otherwise specified) chemicals were used in this work as provided, without further purification. The aqueous solutions were prepared with deionized water (DIW). The chemical products, including the DIW, were purchased from VWR, Belgium.

The dense array of parallel vertically-standing Ni NTs was produced by electrochemical synthesis within the nanopores of a supported AAO template (Fig. 1). A freshly cleaned Si wafer was first coated with a Pt/Al (50/1000 nm) bi-layer using a magnetron sputtering equipment (Plassys MP500S). An ultrathin Ti film ( $5 \mathrm{~nm}$ ) was also employed between the substrate and the successive metallic layers to act as an adhesion promoter. Although $\mathrm{Au}$ is typically preferred as electrode well-withstanding the electrochemical processes, ${ }^{\text {41,42 }}$ Pt was

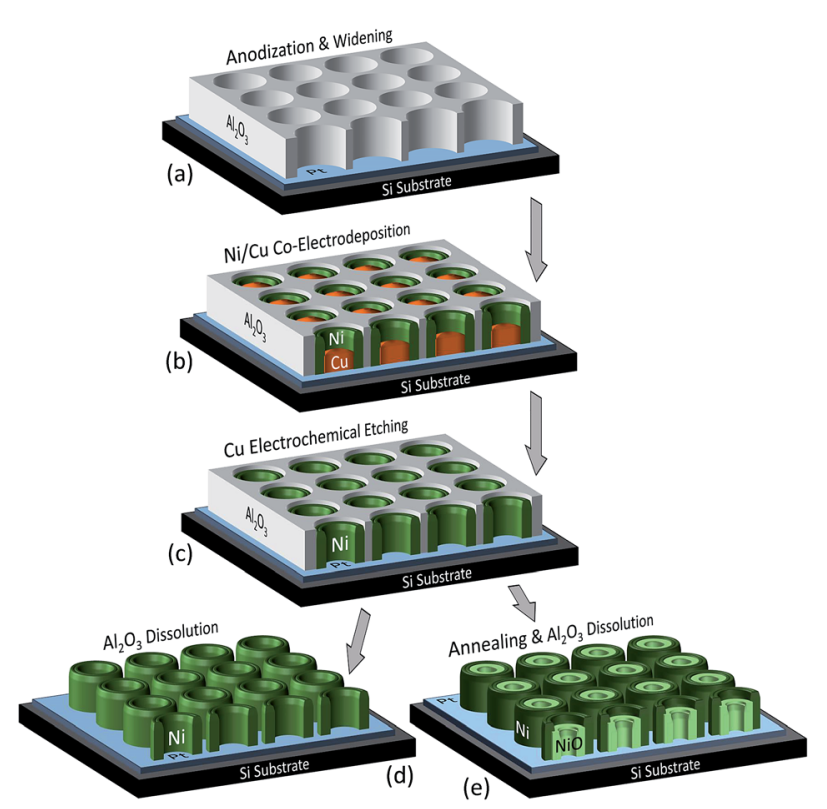

Fig. 1 Schematic representation of the vertically-aligned heterostructured $\mathrm{Ni} / \mathrm{NiO}$ NTs fabrication process. (a) Formation of the thin nanoporous AAO host supported on a Pt-coated Si substrate. (b) $\mathrm{Cu} / \mathrm{Ni}$ core/shell NWs growth by co-ECD. (c) Cu core dissolution by electrochemical etching. (d) AAO film chemical dissolution, revealing $\mathrm{Ni}$ NTs perpendicularly-aligned to the $\mathrm{Pt} / \mathrm{Si}$ substrate. (e) Thermal annealing step followed by AAO dissolution, generating heterostructured Ni/NiO NTs vertically-aligned to the Pt/Si substrate. selected in this work for a better substrate's mechanical and chemical stability during the forthcoming high-temperature annealing treatments.

The as-prepared substrate was next exposed to a single step anodization process in $0.3 \mathrm{M}$ oxalic acid at $2{ }^{\circ} \mathrm{C}$, by applying $60 \mathrm{~V}$ from a Keithley 2400 sourcemeter. The obtained nanopores were then chemically widened in $0.5 \mathrm{M} \mathrm{H}_{2} \mathrm{SO}_{4}$ at $40{ }^{\circ} \mathrm{C}$ for $2 \mathrm{~h}$ [Fig. 1(a)]. The resulting AAO film had a thickness of $\sim 1.4 \mu \mathrm{m}$, as well as a nanopores mean interspacing $(D)$ of $\sim 150 \mathrm{~nm}$ and an average pores diameter $(\phi)$ of $\sim 110 \mathrm{~nm}$, thus a porosity $(P)$ of $\sim 50 \%$ estimated from: ${ }^{16}$

$$
P[\%]=\frac{\pi}{2 \sqrt{3}} \times \frac{\phi^{2}}{D^{2}} \times 100
$$

In a second stage, arrays of $\mathrm{Cu} / \mathrm{Ni}$ core/shell NWs were grown in the pores of the supported AAO template [Fig. 1(b)] by coelectrodeposition (ECD). ${ }^{\mathbf{2 6}, 43}$ The core/shell NWs were cosynthesized at room temperature from an aqueous bath containing: $0.4 \mathrm{M} \mathrm{Ni}\left(\mathrm{H}_{2} \mathrm{NSO}_{3}\right)_{2} \cdot 4 \mathrm{H}_{2} \mathrm{O}, 0.05 \mathrm{M} \mathrm{CuSO}_{4} \cdot 5 \mathrm{H}_{2} \mathrm{O}$ and $0.1 \mathrm{M} \mathrm{H}_{3} \mathrm{BO}_{3}$. The process was carried out in a 'three-electrodes' potentiostatic configuration (using a PAR 263A Potentiostat) by applying to the working electrode (the substrate) $-1 \mathrm{~V}$ versus a double-junction $\mathrm{Ag} / \mathrm{AgCl}$ reference electrode (KCl saturated, $E$ $=0.197 \mathrm{~V}$ ), while a Pt foil was installed in the bath as a counter electrode. The process duration was adjusted to $65 \mathrm{~s}$ for an average length of the AAO-embedded core/shell NWs of $\sim 400 \mathrm{~nm}$. The obtained cathodic reduction curve is depicted in Fig. 2(a), resembling the typical ECD behavior of an AAOassisted chronoamperometric process, where the sharp current increase in the beginning is mainly related with the formation of a diffusion layer of cations in the solution and the NWs nucleation on the Pt cathode at the bottom of the pores, whilst the subsequent current plateau occurs when the gradient of ions concentration is equilibrated and is mainly associated with the NWs vertical growth within the AAO nanopores. The $\mathrm{Cu} / \mathrm{Ni}$ core/shell NWs are obtained in specific conditions (applied potential and concentration ratio between the cations) due to a phase separation between the two metallic elements triggered by an existing miscibility gap in their solubility. Their vertical growth is thus supported by the different nucleation rates between the two phases, where the faster $\mathrm{Cu}$ reduction induces the initial formation of small $\mathrm{Cu}$ islands, while the $\mathrm{Ni}$ growth is progressively enhanced at the islands periphery as the solution is depleted from $\mathrm{Cu}$ cations.

Next, the $\mathrm{Cu}$ cores were electrochemically etched [Fig. 1(c)] using the same bath and configuration, by applying a positive potential of $+0.2 \mathrm{~V}$, while the $\mathrm{Ni}$ did not present a significant etching due to its passivation in sulfamate solutions, ${ }^{43-45}$ ultimately favoring the formation of Ni NTs. Fig. 2(b) shows the obtained oxidation current curve, where the sharp initial negative increase is attributed to the large amount of available $\mathrm{Cu}$. Once the $\mathrm{Cu}$ anions concentration in the solution increases, the amount of solid $\mathrm{Cu}$ inside the Ni NTs reduces and the oxidation rate decreases determining the current to progressively approach zero once the $\mathrm{Cu}$ dissolution is finished. ${ }^{46}$ 

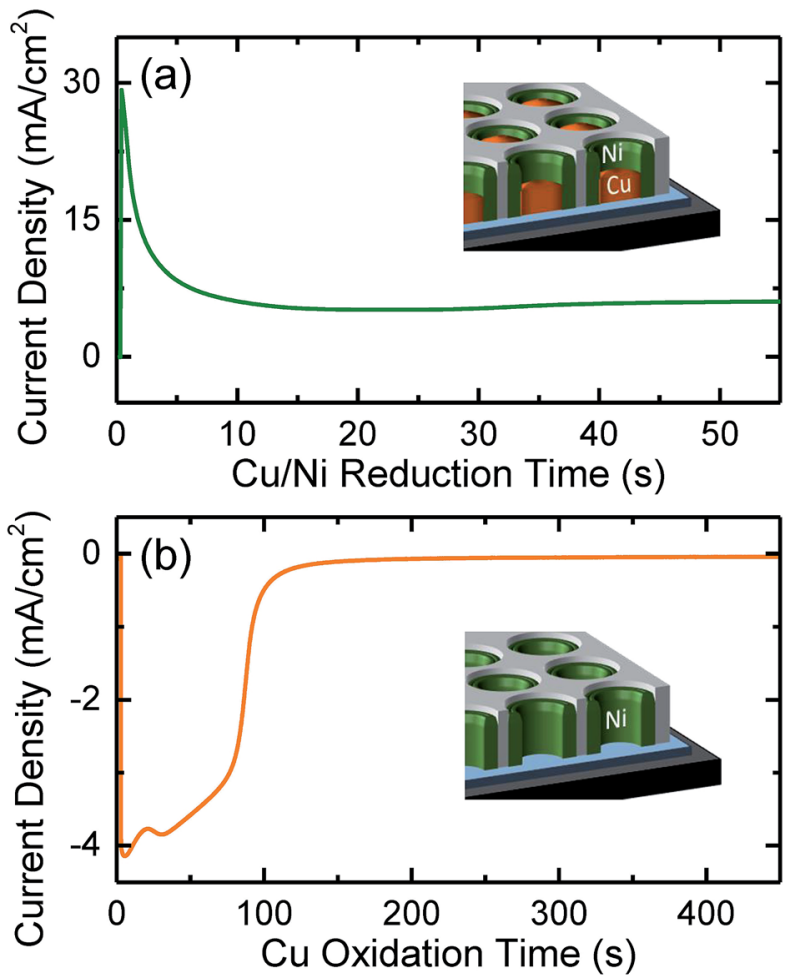

Fig. 2 (a) Typical curve showing the cathodic reduction during the coECD of $\mathrm{Cu} / \mathrm{Ni}$ core/shell NWs embedded within the supported AAO template. (b) Corresponding oxidation curve for the Cu core etching step. The insets show the two equivalent fabrication steps, as detailed in the text.

Noteworthy, the template synthesis confinement plays an important role in the formation of such coaxial $\mathrm{Cu} / \mathrm{Ni}$ architectures with easily removable $\mathrm{Cu}$ cores. In particular, the coECD within nanopores as small as $50 \mathrm{~nm}$ determines the formation of complex mixed-phase NWs made up of a $\mathrm{Cu}-\mathrm{Ni}$ alloy, ${ }^{44}$ as observed also in the case of thin films co-deposited electrochemically by template-free approaches where a subsequent $\mathrm{Cu}$ etching step triggers the occurrence of interesting $\mathrm{Ni}$ nodular structures with hollow cavities..$^{35}$

Thermal treatments were ultimately performed using a Carbolite GTF 12/728 tube furnace in order to convert the subsequently obtained Ni NTs into heterostructured Ni/NiO NTs. The annealing steps were performed without removing the AAO host for preventing NTs outer radial expansion during the thermal oxidation stress, whilst the hollow tubular structure of the $\mathrm{Ni}$ nanoarchitectures allowed their inner thermal exposure. The samples were ramped with $5{ }^{\circ} \mathrm{C} \min ^{-1}$ at an annealing temperature of $450{ }^{\circ} \mathrm{C}$, kept there for $30 \mathrm{~min}, 105 \mathrm{~min}$ and $240 \mathrm{~min}$, respectively, and finally taken out from the oven and left to slowly cool down to room temperature. ${ }^{47}$

The AAO nanoporous host was optionally removed [Fig. 1(d) and (e)], before or after the thermal annealing procedures, mainly to allow subsequent morphological observations of the NTs. The AAO chemical etching was performed at room temperature in a $2 \mathrm{M} \mathrm{NaOH}$ solution for $15 \mathrm{~min}$. The samples were afterwards carefully and thoroughly rinsed with DIW and then left to dry under atmospheric conditions for several hours. ${ }^{40}$

\subsection{Samples characterization}

Morphological observations of the samples were performed using a JEOL $7600 \mathrm{~F}$ field-emission scanning electron microscope (SEM), equipped with transmission electron microscopy (STEM) capabilities. The STEM specimen was prepared by scratching the Ni NTs from the substrate and spreading them on a typical mesh support using tiny isopropanol droplets.

Structural characterization of the NTs before and after the thermal conversion step was accomplished by using an X-ray diffraction (XRD) equipment operating with $\mathrm{CuK \alpha} \alpha_{1}$ radiation $(\lambda=1.54056 \AA)$.

Magnetic investigations were carried out by alternating gradient magnetometery (AGM), using a PMC MicroMag 2900 apparatus. Hysteresis loops and First-Order Reversal Curves (FORCs) were consequently obtained at room temperature with the magnetic field applied parallel and/or perpendicular to the NTs axis.

\section{Results and discussion}

\subsection{Morpho-structural investigation}

Fig. 3 shows the SEM top-view micrographs of the electrochemically-grown NTs, before (a and b) and after (c) a thermal annealing procedure at $450{ }^{\circ} \mathrm{C}$ for $105 \mathrm{~min}$. The images were acquired after the complete dissolution of the AAO embedding template. As can be noticed, before the annealing step a dense array of Ni NTs perpendicularly-arranged to the substrate was obtained [Fig. 2(a) and (b)]. The as-prepared NTs exhibited an outer diameter of $\sim 110 \mathrm{~nm}$ (established by the mean diameter of the pores within the confining AAO template) and an inner diameter of $\sim 80 \mathrm{~nm}$, thus leading to an average NTs wall thickness of $\sim 15 \mathrm{~nm}$, as confirmed by the SEM observations. Next, additional STEM analysis pointed out that the obtained elongated $\mathrm{Ni}$ nanostructures exhibited indeed a homogenous tubular structure being hollow on their whole length [inset of Fig. 3(a)]. The NTs length was adjusted by mainly controlling the accumulated charge during the co-ECD process, at values around $400 \mathrm{~nm}$. In addition, after the annealing step [Fig. 3(c)] the overall aspect morphology of the NTs was similar with the one observed before the annealing process. However, a slight reduction of the NTs inner diameter of several $\mathrm{nm}$ can be observed, whereas the outer diameter remained consistent with the result observed before the thermal treatment. The latter observation was attributed to the presence of the AAO host preventing the radial outer expansion of the NTs during the annealing procedure. Moreover, due to the very small initial wall thickness of the Ni NTs, the annealed $\mathrm{Ni} / \mathrm{NiO}$ heterostructures remained opened, still resembling a tubular structure, although small irregularities seen in the inner diameter might suggest inhomogeneous oxidation of the initial Ni NTs wall. Under similar conditions, it was demonstrated elsewhere that the complete closure of the heterostructures wall might be reached for a total annealing duration of about 300 min. $^{36}$

Fig. 4 shows the XRD spectra acquired from two substrates with vertically-aligned Ni NTs as-prepared (a) and heterostructured 

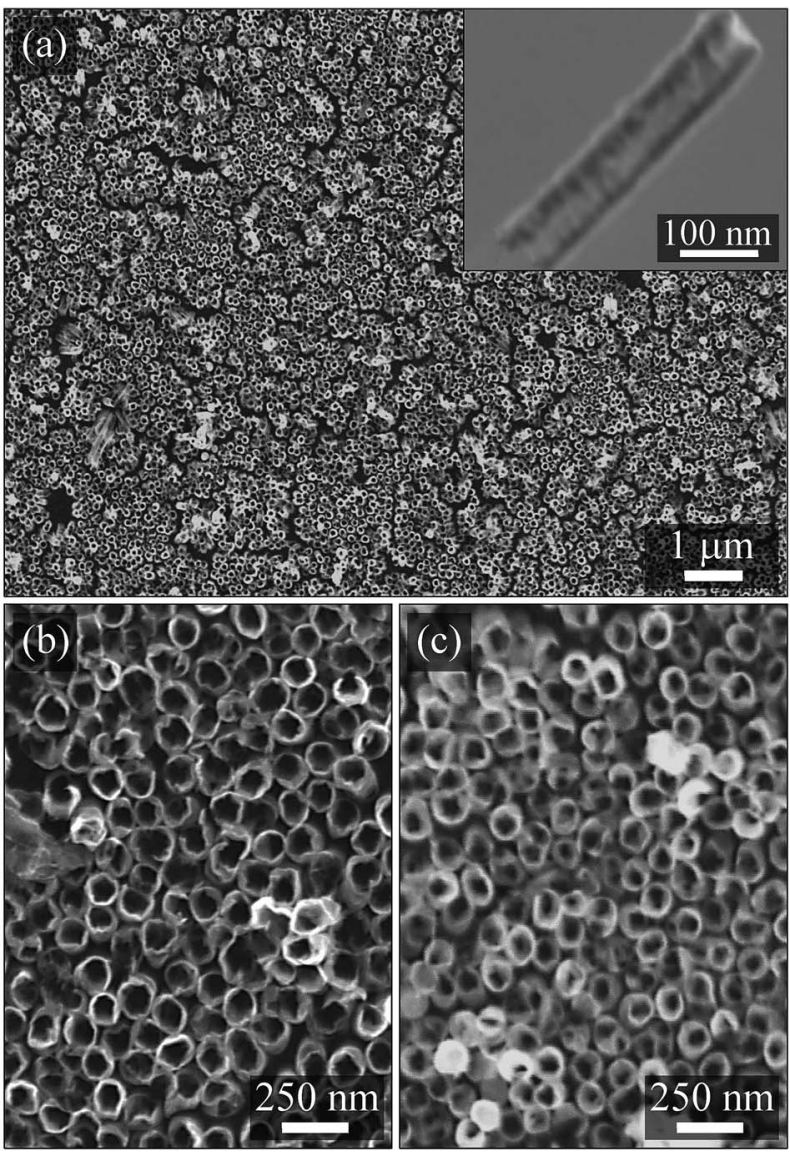

Fig. 3 ( $a$ and b) SEM images at two different magnifications of the dense array of Ni NTs vertically-aligned on the substrate. The inset of (a) STEM image of a single Ni NT as-deposited on the specimen mesh. (c) Corresponding SEM image of the heterostructured $\mathrm{Ni} / \mathrm{NiO} \mathrm{NTs}$ obtained after an annealing treatment at $450{ }^{\circ} \mathrm{C}$ for $105 \mathrm{~min}$.

$\mathrm{Ni} / \mathrm{NiO}$ NTs after annealing at $450{ }^{\circ} \mathrm{C}$ for $105 \mathrm{~min}$ (b), respectively. The typical Pt diffraction peaks at $\sim 39.5^{\circ}$ and $\sim 46^{\circ}$ corresponding to (111) and (200) Miller indices (JCPDS 04-0802), were observed in both situations being definitely attributed to the substrate. Despite

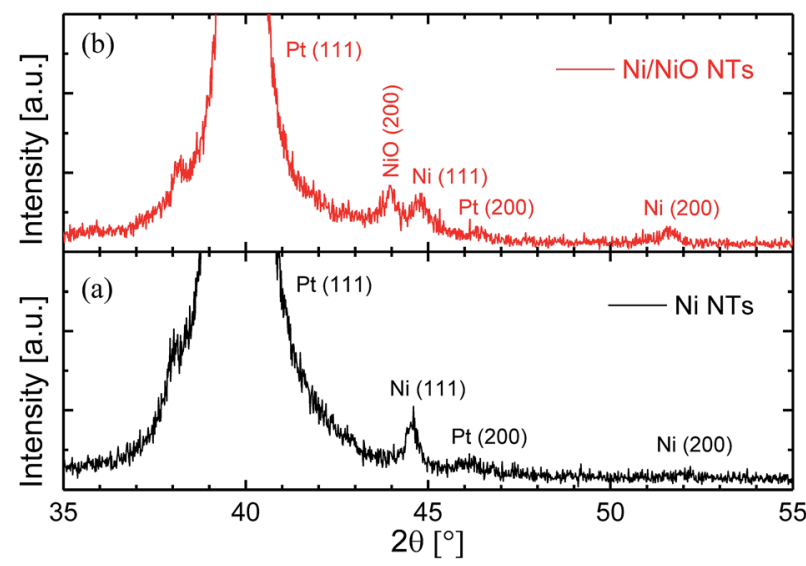

Fig. 4 XRD pattern of the NTs vertically-aligned to the substrate, before (a) and after (b) the annealing process performed at $450{ }^{\circ} \mathrm{C}$ for $105 \mathrm{~min}$. the relatively broad and predominant Pt (111) peak, the common metallic Ni (111) phase at $\sim 44.5^{\circ}$ was clearly observed on both non-annealed and annealed samples, although the additional $\mathrm{Ni}$ (200) peak at $\sim 52^{\circ}$ was slightly enhanced only after the annealing process (JCPDS 01-1260), possibly due to $\mathrm{Ni}$ thermal recrystallization mechanisms, as reported elsewhere. ${ }^{18,48}$ Further- $^{-1}$ more, the annealing process complementarily generated a diffraction peak at $\sim 43.5^{\circ}$ attributed to the $\mathrm{NiO}(200)$ crystal phase (JCPDS 22-1189) and thus demonstrating the partial conversion of the Ni NTs into heterostructured Ni/NiO NTs. The absence of the $\mathrm{NiO}(111)$ peak around $38^{\circ}$ could be particularly explained by the presence of the broad Pt (111) peak within the XRD spectra. The obtained results can be also corroborated with the magnetic measurements presented in Section 3.2, that ultimately confirmed the formation of hybrid Ni/NiO NTs via the thermal annealing approach.

\subsection{Magnetic characterization}

Fig. 5(a) shows the effect of annealing for the various samples on the hysteresis loops obtained at room temperature with the field applied parallel (\|) to the NTs axis. The data were normalized to the measured saturation magnetic moment for the non-annealed (reference) sample. In comparison with the reference sample, the annealed samples have shown a significant decrease of the saturation magnetic moment, to about $53 \%, 39 \%$ and $12 \%$ of the initial value for the samples annealed during $30 \mathrm{~min}, 105 \mathrm{~min}$ and $240 \mathrm{~min}$, respectively. Such evolution was due to the progressive oxidation of the Ni NTs during the annealing process. Fig. 5(b) and (c) compare the room temperature hysteresis loops along $\|$ and perpendicular $(\perp)$ direction to the NTs axis, for the reference sample and for the sample annealed during $105 \mathrm{~min}$. It was found that the nonannealed sample exhibited an easy plane of magnetization, with a normalized remanent magnetization (squareness) $M_{\mathrm{r}} / M_{\mathrm{s}}$ of 0.19 and 0.33 , as well as coercive fields $\left(H_{\mathrm{c}}\right)$ of 160 Oe and 180 Oe, for the field applied $\|$ and $\perp$ to the NTs axis, respectively [see Fig. 5(b)]. Subsequently, the annealing process led to a change in easy axis from $\perp$ to $\|$ to the NTs axis, as illustrated for the sample annealed during $105 \mathrm{~min}$. Along the NTs axis, $M_{\mathrm{r}} /$ $M_{\mathrm{S}}$ increased from 0.19 up to 0.53 and the coercive field also raised from 160 Oe up to 310 Oe [see Fig. 5(c)]. The same qualitative behavior was found for the sample annealed during $30 \min \left(M_{\mathrm{r}} / M_{\mathrm{s}}=0.4\right.$ and $\left.H_{\mathrm{c}}=310 \mathrm{Oe}\right)$. In contrast, the annealing caused no appreciable change in the shape of the hysteresis loops along the $\perp$ direction. Therefore, the annealing process led to an increase in the effective shape anisotropy as evidenced by the less sheared hysteresis loops along the $\|$ direction to the NTs axis [see Fig. 5(a)], thus reinforcing the latter as the easy axis. For an assembly of high aspect ratio nanoparticles, it is known that the effective anisotropy results from the anisotropy of the particle lowered by the DIF between them, i.e. antiferromagnetic interaction in the case of parallel arrays of NTs. ${ }^{26,49}$ Since the shape anisotropy in such elongated nanomagnets is not modified by the annealing process, the enhancement of the shape anisotropy can be then attributed to a decrease of the DIF, which is known to directly depend on the 

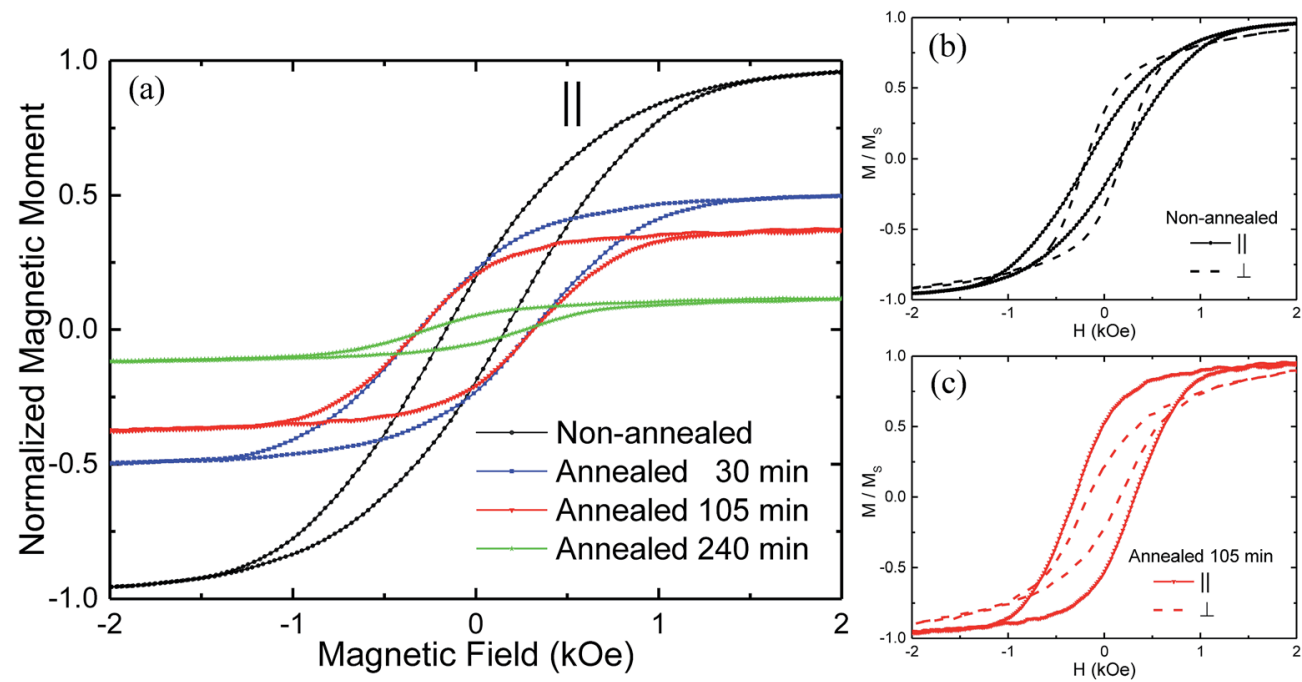

Fig. 5 (a) Typical hysteresis loops measured along parallel (\|) direction to the NTs axis, showing the decrease of the saturation magnetic moment with the annealing time. The data were normalized to the measured saturation magnetic moment for the non-annealed (reference) sample. (b and c) Corresponding hysteresis loops acquired parallel ( $\|$ ) and perpendicular ( $\perp$ ) to the NTs axis for the reference sample (b) and for the sample annealed at $450^{\circ} \mathrm{C}$ for a duration of $105 \mathrm{~min}$ (c).

effective filling factor of particles in the array. ${ }^{49}$ Furthermore, since the distance between NTs and outer diameter remained constant during the annealing process, the reduction of the DIF along the NTs axis and consequently the enhancement of the shape anisotropy ruled out the hypothesis of the uniform oxidation of the Ni NTs. On the contrary, our results are consistent with an oxidation of the inner surface of the NTs which results in a reduction of the wall thickness of the nonoxidized Ni NTs in contact with the pore walls of the AAO template. Indeed, it was demonstrated both theoretically and experimentally that the cavities in NT assemblies reduces the DIF when compared with NW assemblies and the derived expressions also predicted that the DIF is further reduced as the wall thickness of the ferromagnetic NTs becomes smaller. ${ }^{25,26}$ Such a scenario involving the growth of heterostructured $\mathrm{Ni} /$ NiO NTs through annealing was consistent with the observed magnetic behavior in the present study.

In order to provide quantitative estimation of the DIF changes during all stages of the annealing process, the AGM was employed to measure a large number (about 150) of FORCs along the NTs axis. In this context, after saturation the magnetization $(M)$ was measured starting from a reversal field $\left(H_{\mathrm{r}}\right)$ back to the positive saturation, tracing out a FORC referred as $M\left(H, H_{\mathrm{r}}\right)$. A family of FORCs was then measured at different $H_{\mathrm{r}}$, with an equal field spacing, thus filling the interior of the major hysteresis loop, as shown in Fig. 6(a)-(c) for the three different NTs array samples. The FORC diagrams were calculated by a mixed second order derivative, as proposed by R. Pike: ${ }^{50}$

$$
\rho\left(H, H_{\mathrm{r}}\right)=-\frac{\partial^{2} M\left(H, H_{\mathrm{r}}\right)}{\partial H \partial H_{\mathrm{r}}}
$$

where $H$ was the applied field and $H_{\mathrm{r}}$ was the return field. The coercive field distribution and the DIF at saturation between particles were obtained by plotting the FORC diagrams in the interaction-coercivity plane. It was thus convenient to change the coordinates to the coercive field $H_{\mathrm{c}}=\left(H-H_{\mathrm{r}}\right) / 2$ and the DIF $H_{\mathrm{u}}=\left(H+H_{\mathrm{r}}\right) / 2$, with $H_{\mathrm{c}}$ and $H_{\mathrm{u}}$ on the horizontal and vertical axes, respectively. For arrays of elongated particles like the NTs, the FORC diagrams can be interpreted properly in a mean-field approximation where the total magnetization dependent DIF is given by $H_{\text {int }}=\alpha_{\mathrm{Z}} m$, with $\alpha_{\mathrm{Z}}$ as the mean DIF factor and $m$ the normalized magnetization which varies between -1 and $+1 .^{51}$ Fig. 6(d)-(f) show the FORC diagrams of the Ni NT arrays embedded in the AAO templates before and after two annealing steps. The FORCs were consistent with the results of the major hysteresis loops shown in Fig. 5(a). Indeed, the maximum observed in the FORCs along the $H_{\mathrm{c}}$ axis can be associated to a good approximation with the average value of the coercive field, i.e. $H_{\mathrm{c}}=200$ Oe for the non-annealed sample and $H_{\mathrm{c}}=360$ Oe for the two annealed samples. Furthermore, for demagnetizing type interactions, the average DIF at saturated state was determined by the half-width of the FORCs distribution along the $H_{\mathrm{u}}$ axis. ${ }^{52-54}$ Rough estimates of the DIF were indicated by black vertical arrows in Fig. 6(d)-(f) for the different NT arrays. It appeared that the DIF factor $\alpha_{\mathrm{Z}}$ was reduced from about 700 Oe for the non-annealed sample to about 400 Oe and 300 Oe after annealing during $30 \mathrm{~min}$ and $105 \mathrm{~min}$, respectively. As discussed before, such a reduction in the DIF is consistent with the less sheared hysteresis loops observed for the annealed samples along $\|$ direction to the NTs axis [see Fig. 5(a)]. The FORC diagrams also show that the reversible magnetization switching was considerably reduced in the annealed NT samples, as evidenced by a strong reduction in the $H_{\mathrm{c}}=0$ component in the measured coercivity distribution. The DIF coefficient $\alpha_{\mathrm{Z}}$ could be estimated using: ${ }^{26,55}$

$$
\alpha_{\mathrm{Z}}=N_{\mathrm{x}} M_{\mathrm{s}} P_{\mathrm{NT}}
$$

where $M_{\mathrm{s}}$ was the saturation magnetization, $N_{\mathrm{x}}$ was the demagnetizing factor of the cylindrical NTs, $P_{\mathrm{NT}}=P\left(1-\beta^{2}\right)$ was 

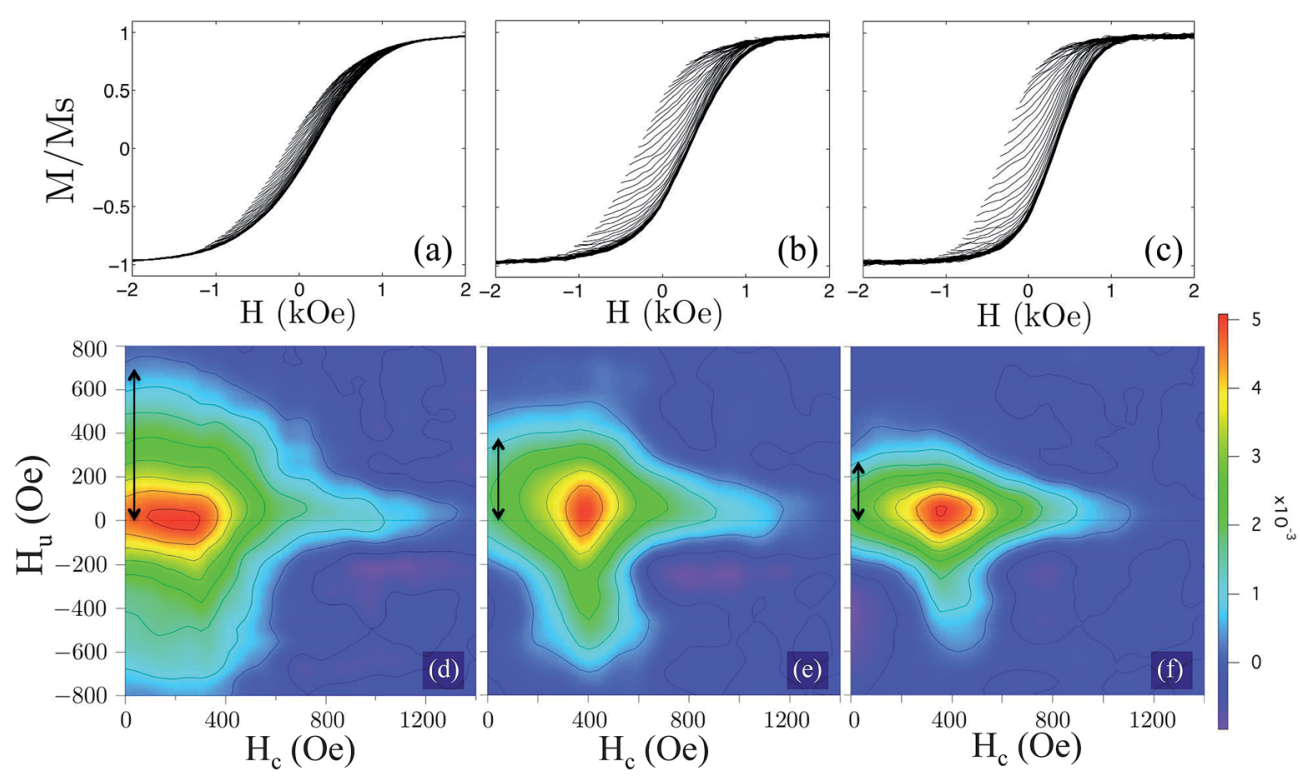

Fig. $6(a-c)$ Magnetization curves and $(d-f)$ corresponding First-Order Reversal Curves (FORCs) obtained for the Ni NT arrays when applying the magnetic field $\|$ to the NTs axis, for the reference sample ( $a$ and d), as well as after annealing durations of 30 min (b and e) and 105 min (c and f) at $T=450^{\circ} \mathrm{C}$. The vertical arrows in the FORC diagrams indicate the magnitude of the dipolar interaction field (DIF), as detailed in the text.

the packing fraction of the NTs, $P$ was the porosity of the nanoporous AAO template, and $\beta=r_{1} / r_{2}$ was the ratio of the internal and external radii of the NTs. ${ }^{26}$ For the non-annealed sample, using known values of $M_{\mathrm{s}(\mathrm{Ni})}=485 \mathrm{emu} \mathrm{cm}^{-3}$ and template porosity $P \sim 50 \%$, taking $N_{\mathrm{x}} \sim 6.02$ for such nanocylinders (with an aspect-ratio, length/diameter of $\sim 3),{ }^{26}$ and using the estimated values of the external and internal diameter of $110 \mathrm{~nm}$ and $80 \mathrm{~nm}$, respectively, as obtained from the SEM analysis, we calculated an $\alpha_{\mathrm{Z}}$ of $\sim 730$ Oe. Next, it was assumed that the reduction of the saturation magnetic moment after annealing shown in Fig. 5(a) was due to the thin metal oxide layer formed on the inner surface of the NTs which consequently led to a decrease of the wall thickness of the metallic Ni NTs. Under this assumption, we could calculate the reduction in the packing fraction of ferromagnetic material $\left(P_{\mathrm{NT}}\right)$ from $25 \%$ for the nonannealed sample to $\sim 13 \%$ and $\sim 10 \%$ after annealing during $30 \mathrm{~min}$ and $105 \mathrm{~min}$, respectively. This led to a reduction of the DIF coefficient $\alpha_{\mathrm{Z}}$ to 387 Oe and 286 Oe after annealing during $30 \mathrm{~min}$ and $105 \mathrm{~min}$, respectively, in great accordance with the values estimated from the FORC diagrams (see Fig. 6). Thus a very good agreement was observed between the measured and calculated values of $\alpha_{\mathrm{z}}$, which shows that both approaches provided correct results for the DIF. In addition, such analysis could be used to estimate the reduction in the ferromagnetic wall thickness from $15 \mathrm{~nm}$ for the non-annealed sample to about $8 \mathrm{~nm}$ and $6 \mathrm{~nm}$ after annealing during $30 \mathrm{~min}$ and $105 \mathrm{~min}$, respectively. Finally, it was noted that when the annealing time was increased to $240 \mathrm{~min}$, both coercivity and squareness were reduced, as shown in Fig. 5(a). Such a behavior was attributed to the fact that the tubular shape of the Ni material was lost due to the long annealing time, leading to an almost complete oxidation, thus giving rise to a discontinuous Ni layer or aggregates in the Ni/NiO NT arrays along the NTs axis.

\section{Conclusions}

In this work we developed an efficient and cost-effective pathway in which large and dense ferromagnetic arrays of vertically-aligned heterostructured $\mathrm{Ni} / \mathrm{NiO}$ NTs can be produced. The method allowed the fabrication of hybrid hollow elongated nanostructures without compromising their verticality to the substrate, as well as their initial tubular integrity. Using adequately adapted AGM measurements, we demonstrated the ability to tune the DIF value within the magnetic array, just by slightly changing the thickness of the antiferromagnetic NiO thermally-grown layer. Furthermore, the subsequent FORCs-based analysis permitted an estimation of the DIF coefficients which were in close agreement with the calculated values among the thermally annealed samples, proving further the hypothesis of retaining a tubular hybrid structure of the $\mathrm{Ni} /$ NiO NTs after the thermal conversion process of the AAOembedded Ni NTs. Such dense arrays of ferromagnetic NTs may therefore potentially withstand individual magnetic addressability, by carefully tuning the DIF value in terms of changing the magnetic packing fraction either by adjusting the template porosity, or ultimately by controlling the degree of NTs thermal conversion step. The entire fabrication protocol is quite promising and amiable to further optimisations, opening an outstanding way towards direct integrability of devices relying on large magnetic arrays of vertically-standing elongated nanostructures.

\section{Acknowledgements}

This work was partly supported by the 'Fédération WallonieBruxelles' (ARC 13/18-052, Supracryst) and by the 'Fonds de la Recherche Scientifique' - FNRS under Grant No. T.0006.16. The 
authors thank Mrs Anne Iserentant (Earth and Life Institute, Université Catholique de Louvain) and Prof. Lucian Ion (Faculty of Physics, University of Bucharest) for the XRD measurements and data interpretation.

\section{References}

1 L. Vila, P. Vincent, L. D.-D. Pra, G. Pirio, E. Minoux, L. Gangloff, S. Demoustier-Champagne, N. Sarazin, E. Ferain, R. Legras, L. Piraux and P. Legagneux, Nano Lett., 2004, 4, 521-524.

2 H. Qi, O. J. Glembocki and S. M. Prokes, J. Nanomater., 2012, 2012, 7 .

3 A. Koka, Z. Zhou and H. A. Sodano, Energy Environ. Sci., 2014, 7, 288-296.

4 A. Koka and H. A. Sodano, Nat. Commun., 2013, 4, 2682.

5 E. A. Anyebe, A. M. Sanchez, S. Hindmarsh, X. Chen, J. Shao, M. K. Rajpalke, T. D. Veal, B. J. Robinson, O. Kolosov, F. Anderson, R. Sundaram, Z. M. Wang, V. Falko and Q. Zhuang, Nano Lett., 2015, 15, 4348-4355.

6 K. M. Razeeb, M. Jamal, M. Hasan and A. Mathewson, Nanobiosensors and Nanobioanalyses, Springer, Japan, Tokyo, 2015, pp. 23-40.

7 T. Berthing, C. B. Sørensen, J. Nygård and K. L. Martinez, J. Nanoneurosci., 2009, 1, 3-9.

8 B. Sanz, E. M. Palmero, R. P. del Real, M. Vázquez and C. Mijangos, Ind. Eng. Chem. Res., 2015, 54, 13005-13008.

9 W. B. Choi, D. S. Chung, J. H. Kang, H. Y. Kim, Y. W. Jin, I. T. Han, Y. H. Lee, J. E. Jung, N. S. Lee, G. S. Park and J. M. Kim, Appl. Phys. Lett., 1999, 75, 3129-3131.

10 H. Chen, A. Roy, J. B. Baek, L. Zhu, J. Qu and L. Dai, Mater. Sci. Eng., $R$, 2010, 70, 63-91.

11 A. Z. Liao, C. W. Wang, J. B. Chen, X. Q. Zhang, Y. Li and J. Wang, Mater. Res. Bull., 2015, 70, 988-994.

12 T. Stergiopoulos, A. Ghicov, V. Likodimos, D. S. Tsoukleris, J. Kunze, P. Schmuki and P. Falaras, Nanotechnology, 2008, 19, 5602 .

13 H. Cao, L. Wang, Y. Qiu, Q. Wu, G. Wang, L. Zhang and X. Liu, ChemPhysChem, 2006, 7, 1500-1504.

14 Y. Zhong, H. Fan, L. Chang, H. Shao, J. Wang, J. Zhang and C. Cao, J. Power Sources, 2015, 296, 255-260.

15 S. J. Son, J. Reichel, B. He, M. Schuchman and S. B. Lee, J. Am. Chem. Soc., 2005, 127, 7316-7317.

16 K. Nielsch, F. J. Castaño, S. Matthias, W. Lee and C. A. Ross, Adv. Eng. Mater., 2005, 7, 217-221.

17 K. Pitzschel, J. Bachmann, J. M. Montero-Moreno, J. Escrig, D. Görlitz and K. Nielsch, Nanotechnology, 2012, 23, 495718.

18 A. Vlad, M. Mátéfi-Tempfli, V. A. Antohe, S. Faniel, N. Reckinger, B. Olbrechts, A. Crahay, V. Bayot, L. Piraux, S. Melinte and S. Mátéfi-Tempfli, Small, 2008, 4, 557-560.

19 A. Vlad, V. A. Antohe, J. M. Martinez-Huerta, E. Ferain, J. F. Gohy and L. Piraux, J. Mater. Chem. A, 2016, 4, 16031607.

20 M. Sharma, B. K. Kuanr, V. Veerakumar, A. Basu and Z. J. Celinski, IEEE Trans. Magn., 2014, 50, 1-4.

21 R. Sharif, S. Shamaila, M. Ma, L. D. Yao, R. C. Yu, X. F. Han and M. K. ur Rahman, Appl. Phys. Lett., 2008, 92, 2505.
22 P. Landeros, S. Allende, J. Escrig, E. Salcedo, D. Altbir and E. E. Vogel, Appl. Phys. Lett., 2007, 90, 2501.

23 X. F. Han, S. Shamaila, R. Sharif, J. Y. Chen, H. R. Liu and D. P. Liu, Adv. Mater., 2009, 21, 4619-4624.

24 A. Pereira, J. L. Palma, J. C. Denardin and J. Escrig, Nanotechnology, 2016, 27, 345709.

25 M. P. Proenca, C. T. Sousa, J. Escrig, J. Ventura, M. Vazquez and J. P. Araujo, J. Appl. Phys., 2013, 113, 3907.

26 Y. Velázquez-Galván, J. M. Martínez-Huerta, J. D. L. T. Medina, Y. Danlée, L. Piraux and A. Encinas, J. Phys.: Condens. Matter, 2014, 26, 6001.

27 V. Skumryev, S. Stoyanov, Y. Zhang, G. Hadjipanayis, D. Givord and J. Nogués, Nature, 2003, 423, 850-853.

28 K. Yadav, B. R. Mehta and J. P. Singh, J. Mater. Chem. C, 2014, 2, 6362-6369.

29 R. S. Chen, H. M. Chang, Y. S. Huang, D. S. Tsai, S. Chattopadhyay and K. H. Chen, J. Cryst. Growth, 2004, 271, 105-112.

30 G. Malandrino, L. M. S. Perdicaro, I. L. Fragalà, R. L. Nigro, M. Losurdo and G. Bruno, J. Phys. Chem. C, 2007, 111, 32113215.

31 S. H. Yu, J. Shin, J. J. Kim, K. J. Lee and Y. E. Sung, RSC Adv., 2012, 2, 12177-12181.

32 H. M. Ouyang, G. T. Fei, Y. Zhang, H. Su, Z. Jin, S. H. Xu and L. D. Zhang, J. Mater. Chem. C, 2013, 1, 7498-7506.

33 J. Choi, J. H. Lim, J. Lee and K. J. Kim, Nanotechnology, 2007, 18, 055603.

34 K. Xie, M. Guo, H. Huang and Y. Liu, Corros. Sci., 2014, 88, 66-75.

35 X. Dai, D. Chen, H. Fan, Y. Zhong, L. Chang, H. Shao, J. Wang, J. Zhang and C. n. Cao, Electrochim. Acta, 2015, 154, 128-135.

36 F. I. Dar, K. Moonoosawmy and M. Es-Souni, Nanoscale Res. Lett., 2013, 8, 363.

37 J. Bao, C. Tie, Z. Xu, Q. Zhou, D. Shen and Q. Ma, Adv. Mater., 2001, 13, 1631-1633.

38 F. Tao, M. Guan, Y. Jiang, J. Zhu, Z. Xu and Z. Xue, Adv. Mater., 2006, 18, 2161-2164.

39 C. Mu, Y. X. Yu, R. M. Wang, K. Wu, D. S. Xu and G. L. Guo, Adv. Mater., 2004, 16, 1550-1553.

40 V. A. Antohe, A. Radu, M. Mátéfi-Tempfli, A. Attout, S. Yunus, P. Bertrand, C. A. Duțu, A. Vlad, S. Melinte, S. Mátéfi-Tempfli and L. Piraux, Appl. Phys. Lett., 2009, 94, 3118.

41 F. A. Araujo, L. Piraux, V. A. Antohe, V. Cros and L. Gence, Appl. Phys. Lett., 2013, 102, 2402.

42 L. Piraux, K. Renard, R. Guillemet, S. Mátéfi-Tempfli, M. Mátéfi-Tempfli, V. A. Antohe, S. Fusil, K. Bouzehouane and V. Cros, Nano Lett., 2007, 7, 2563-2567.

43 Q. Wang, G. Wang, X. Han, X. Wang and J. G. Hou, J. Phys. Chem. B, 2005, 109, 23326-23329.

44 Z. Liu, G. Xia, F. Zhu, S. Kim, N. Markovic, C. L. Chien and P. C. Searson, J. Appl. Phys., 2008, 103, 4313.

45 Z. Liu, D. Elbert, C. L. Chien and P. C. Searson, Nano Lett., 2008, 8, 2166-2170.

46 J. K. Chang, S. H. Hsu, I. W. Sun and W. T. Tsai, J. Phys. Chem. C, 2008, 112, 1371-1376. 
47 S. Han, H. Y. Chen, C. C. Chen, T. N. Yuan and H. C. Shih, Mater. Lett., 2007, 61, 1105-1108.

48 L. D. L. S. Valladares, A. Ionescu, S. Holmes, C. H. W. Barnes, A. B. Domínguez, O. A. Quispe, J. C. González, S. Milana, M. Barbone, A. C. Ferrari, H. Ramos and Y. Majima, J. Vac. Sci. Technol., B: Nanotechnol. Microelectron.: Mater., Process., Meas., Phenom., 2014, 32, 051808.

49 A. Encinas-Oropesa, M. Demand, L. Piraux, I. Huynen and U. Ebels, Phys. Rev. B: Condens. Matter Mater. Phys., 2001, 63, 4415.

50 C. R. Pike, A. P. Roberts and K. L. Verosub, J. Appl. Phys., 1999, 85, 6660-6667.
51 G. Bertotti, in Hysteresis in Magnetism, Academic Press, San Diego, 1998, pp. 129-162.

52 F. Béron, L. Clime, M. Ciureanu, D. Ménard, R. W. Cochrane and A. Yelon, J. Nanosci. Nanotechnol., 2008, 8, 2944-2954.

53 C. I. Dobrotă and A. Stancu, J. Appl. Phys., 2013, 113, 3928.

54 D. A. Gilbert, G. T. Zimanyi, R. K. Dumas, M. Winklhofer, A. Gomez, N. Eibagi, J. L. Vicent and K. Liu, Sci. Rep., 2014, 4, 4204 .

55 J. M. Martínez-Huerta, J. D. L. T. Medina, L. Piraux and A. Encinas, J. Phys.: Condens. Matter, 2013, 25, 6003.

56 R. Cai, V.-A. Antohe, Z. Hu, B. Nysten, L. Piraux and A. M. Jonas, Adv. Mater., 2017, 29, 1604604. 